\title{
28 Research Square \\ Cytokine Biomarker Phenotype for Early Prediction and Triage of Sepsis in Blunt Trauma Patients
}

\section{Jun Wang}

Guizhou Medical University

Dalin Wen

Army Medical University

Shi Zeng

Army Medical University

Juan Du

Army Medical University

Li Cui

Army Medical University

Jianhui Sun

Army Medical University

Guosheng Chen

Guizhou Medical University

Ling Zeng

Army Medical University

Dingyuan Du

Chongqing Emergency Medical Center

Lianyang Zhang

Army Medical University

Jin Deng

Guizhou Medical University

Jianxin Jiang

Army Medical University

Anqiang Zhang ( $\nabla$ zhanganqiang@126.com )

Army Medical University https://orcid.org/0000-0002-0375-2078

Research article

Keywords: trauma, sepsis, early prediction, cytokine, biomarker

Posted Date: February 21st, 2022 
DOl: https://doi.org/10.21203/rs.3.rs-84088/v2

License: (c) (1) This work is licensed under a Creative Commons Attribution 4.0 International License. Read Full License 


\section{Abstract}

Background: The study aimed to establish the profiles of cytokines in trauma patients to characterize the nature of the immune responses to sepsis, which might enable early prediction and individualized treatments to be developed and targeted.

Methods: A 15-plex human cytokine magnetic bead assay system was used to measure analytes in citrated plasma samples. Analysis of these cytokine kinetics was performed on 40 patients with severe blunt trauma admitted to our trauma center between March 2016 and February 2017 with Injury Severity Score (ISS) more than 20 with regard to sepsis (Sepsis-3) over a 14-day time course.

Results: In total, six cytokines changed in trauma patients between the different timepoints at day 1, 3, 5, 7, and 14. Additionally, IL-6, IL-10, IL-15, MDC, GRO, sCD40L, G-CSF, and FGF-2 significantly distinguished between sepsis and nonsepsis at day 3 and afterward with an area under the curve of up to 0.90 when the eight biomarkers $(P<0.001)$ were combined. Event-related analysis demonstrated 1.5 - to 4 -fold serum level changes for these cytokines within 72 hours before clinically apparent sepsis.

Conclusion: Cytokine profiles demonstrate high discriminatory ability to timely identify evolving traumatic sepsis. These abrupt changes allow sepsis detection up to 72 hours before clinically overt deterioration. Measurement of these cytokines might enable future studies to better predict, diagnose, and characterize traumatic sepsis, as well as confer the potential for physicians to timely initiate treatment with reduced mortality and costs.

Trial registration: Clinicaltrials, NCT01713205. Registered 22 October 2012, https://register.clinicaltrials.gov/NCT01713205

\section{Background}

Sepsis is a major public health crisis worldwide. Despite the huge advances in treatment technology, over 5.3 million patients die annually from sepsis, at an estimated overall mortality of $30 \%$ [1]. Sepsis, defined as "life-threatening organ dysfunction caused by a dysregulated host response to infection according to the Third International Consensus Definitions for Sepsis and Septic Shock (Sepsis-3)", is counted among the leading causes of major trauma-related mortality worldwide [2]. But, it has proven difficult to develop clinical and laboratory criteria that accurately predict risk for mortality, particularly in the early period of sepsis. Timely detection and treatment of emerging infections can allow us to take measures for treatment as early as possible, thereby reducing post-trauma mortality and improving patient outcome [3, 4]. Hence, in the setting of imperfect diagnostic and prognostic tools for sepsis, novel methods for effective identification of severe trauma patients who have the potential to develop life-threatening infectious complications and are at the risk of death is still an urgent problem.

Severe trauma could induce exacerbation of systemic inflammation, which often progresses to sepsis leading to a lethal outcome. Inflammatory imbalance represents the most critical basis of sepsis 
pathogenesis and occurs throughout the whole process of sepsis, and the pathogens eliciting the response include organisms such as bacteria, fungi, parasites, and viruses [5]. The host's initial acute response to invasive pathogens typically causes macrophages to engulf the pathogens and produce a range of pro- and anti-inflammatory cytokines, and this can trigger cytokine storms and activate the immune system [6]. Numerous evidence [7] has been sought to identify effective biomarkers to predict which patients are at high-risk of morbidity and mortality. Many biomarkers [8,9], such as C-reaction protein (CRP), procalcitonin (PCT), tumor necrosis factor (TNF)-a, interleukin-6 (IL-6), and IL-8, which are involved in systemic inflammation caused by trauma and relate to the severity of injury, have been assessed as potential markers to predict poor prognosis in critical ill patients. Extensive data are also available for sepsis-related changes in macrophage inflammatory protein (MIP)- $1 \alpha / \beta$, heat shock protein (HSP) 70, matrix metalloprotease (MMP)-8, and granzyme B in animal or adult models [7]. Unfortunately, all of these biomarkers lack sufficient specificity. In addition, various rule-based disease-severity scoring systems are also widely used in an attempt to identify sepsis patients. These scores, such as the Systemic Inflammatory Response Syndrome (SIRS) criteria [10], the Modified Early Warning Score (MEWS) [11], and the Sequential Organ Failure Assessment (SOFA) [12], are manually tabulated at the bedside and lack accuracy in sepsis diagnosis.

Therefore, improved biomarkers are required for the prompt diagnosis of sepsis and prediction of outcomes. However, previous reports have often evaluated individual biomarkers. In this study, inflammatory biomarkers, including IL-6, IL-8, IL-10, IL-15, TNF-a, macrophage derived chemokine (MDC), interferon-inducible protein (IP)-10, eotaxin, soluble CD40 ligand (sCD40L), growth regulated oncogene (GRO), monocyte chemotactic protein (MCP)-1, MIP-1 $\beta$, fibroblast growth factor (FGF)-2, granulocyte colony-stimulating factor (G-CSF) and granulocyte macrophage colony-stimulating factor (GM-CSF) were analyzed at five timepoints $(1,3,5,7$, and 14 days after trauma) in forty severe trauma patients. The objective of the present study was to investigate the time course of these cytokine levels in patients with severe trauma and to determine whether cytokines can serve as a biomarker to predict the development of sepsis in trauma patients.

\section{Methods}

\section{Study population}

This study was conducted with data obtained at the Department of Trauma Surgery in Daping Hospital and the Chongqing Emergency Medical Center from March 2016 to February 2017. The inclusion criteria were the following: (1) the age of patients is between 18 and 65 years, (2) ISS is greater than 20, and (3) patients survive for longer than 48 hours after injury. Patients were excluded if they had penetrating injuries, preexisting organ dysfunction, or immune diseases. This study was approved by the Ethical and Protocol Review Committees of Army Medical University and followed the guidelines of the Declaration of Helsinki. Informed consent was obtained from the patients and their next of kin before enrollment, including explicit permission for plasma analysis and the collection of relevant clinical data. 


\section{Clinical Evaluation}

Demographic characteristics and laboratory and clinical data were collected by expert physicians. The ISS was calculated according to the Abbreviated Injury Scale developed in 2005 [13]. Sepsis was diagnosed based on a rapid change in SOFA score $\geq 2$, with evidence of infection during the hospital period according to "Sepsis-3" [2]. Infection [14] was defined as a clinically obvious source or positive bacterial culture by medical examinations as follows: microbiological tests, including culture of body fluids; conventional or real-time polymerase chain reaction; radiological analyses, including ultrasonography, X-ray, and computed tomography; and serology. SOFA scores [12] were calculated daily as the sum of the simultaneously obtained individual organ scores, including respiratory, cardiovascular, hepatic, renal, coagulation, and neurologic systems.

\section{Multiplex Cytokine Detection}

After informed consent, venous blood samples were drawn on days 1, 3, 5, 7, and 14 during the first 14 days after trauma, encompassing $>90 \%$ of the outcome events. Plasma was separated, aliquoted, and stored at $-80^{\circ} \mathrm{C}$ until analyzed. The multiplex cytokine kits (IL-6, IL-8, IL-10, IL-15, TNF-a, MDC, IP-10, eotaxin, SCD40L, GRO, MCP-1, MIP-1 $\beta$, FGF-2, G-CSF, and GM-CSF) were obtained, and the assay was performed on the Luminex200 system in accordance with the manufacturer's instructions (Millipore, Billerica, MA, USA). The detectable range of each cytokine was 0.89 to $3500 \mathrm{pg} / \mathrm{mL}$. All measurements were made by the same person who was blinded to the clinical data.

\section{Statistical analysis}

Discrete data are expressed as counts with percentages, whereas continuous data are presented as the median with interquartile range (IQR) or the mean \pm standard deviation (SD) as appropriate. Baseline characteristics were compared between groups using the Chi-square test for counts and univariate analysis of variance for continuous data. Biomarker time courses were compared between groups using Wilcoxon's rank sum test. Receiver operating characteristic (ROC) curve analysis was employed at each timepoint to evaluate the predictive performance of the biomarkers tested with regard to traumatic sepsis. Values of the Area Under Curve (AUC) are reported with the corresponding 95\% confidence interval (Cl). An AUC greater than 0.7 was considered good. All tests were 2 tailed; $P<0.05$ was considered significant. Data were analyzed using SPSS (Statistical Package for Social Sciences, Version 22 for Macintosh; SPSS Inc., Chicago, IL) and GraphPad Prism version 5.0 for Macintosh (GraphPad Software, La Jolla CA).

\section{Results}

\section{Clinical characteristics of trauma patients}


Baseline characteristics of the study population in total and according to the main outcome (no sepsis vs. sepsis vs. septic shock) are given in Table 1. Forty severely injured patients ( 9 female) with ISS $\geq 20$ were included. The median periods from injury to admission to the hospital were 5.5 hours (4.0-17.3 hours). We pooled the septic and septic shock group for further analyses due to the low number of patients with septic shock $(n=7)$. A total of 22 patients $(55 \%)$ developed septic complications within the first 14 days. The median timepoint for sepsis occurrence was 4.5 days (2.0-7.0 days). The mean age was $43.25 \pm 13.44$ years and did not differ between the 2 groups $(P=0.059)$. The total median ISS was 29.00 (22.50-34.00) and differed significantly in the subgroup analysis (no sepsis: 24.50 (22.00-33.25), sepsis: 29.00 (28.50-35.75); $P=0.048)$. None of the patients died. Septic patients had a comprehensibly longer stay in the Intensive Care Unit (ICU) than patients with an uneventful course $(P=0.003)$. We focused on the infectious events that occurred within the first 14 days, observing pneumonia in 18 patients (45.0\%), followed by bacteremia in 5 patients (12.5\%), 4 patients with wound infection (10.0\%), and 3 patients $(7.5 \%)$ with urinary tract infection. Pathogen testing revealed that gram-negative bacterial infection occurred in $22.5 \%$ of patients, gram-positive infection in $15 \%$ of patients and mixed-gram infection in $10 \%$ of patients. 
Table 1

Clinical characteristics of trauma patients.

\begin{tabular}{|c|c|c|c|c|}
\hline Variables & Total $(n=40)$ & $\begin{array}{l}\text { Non-sepsis } \\
(\mathrm{n}=18)\end{array}$ & Sepsis $(n=22)$ & $\begin{array}{l}P \\
\text { value }\end{array}$ \\
\hline Age $(y)$, mean $\pm S D$ & $43.25 \pm 13.44$ & $38.83 \pm 14.77$ & $46.86 \pm 11.34$ & 0.059 \\
\hline Sex (female), n (\%) & $9(22.5 \%)$ & $5(27.7 \%)$ & $4(18.2 \%)$ & 0.470 \\
\hline History of comorbidity, n (\%) & $6(15.0 \%)$ & $2(11.1 \%)$ & $4(18.2 \%)$ & 0.533 \\
\hline ISS, median (IQR) & $\begin{array}{l}29.00(22.50- \\
34.00)\end{array}$ & $\begin{array}{l}24.50(22.00- \\
33.25)\end{array}$ & $\begin{array}{l}29.00(28.50- \\
35.75)\end{array}$ & 0.048 \\
\hline ISS>25, n (\%) & $26(65 \%)$ & $8(44.4 \%)$ & $18(81.8 \%)$ & \\
\hline ISS $\leq 25, \mathrm{n}(\%)$ & $14(35 \%)$ & $10(55.6 \%)$ & $4(18.2 \%)$ & \\
\hline $\mathrm{AIS}_{\text {head/neck, }}$ median (IQR) & $3.00(0.00-4.00)$ & $\begin{array}{l}2.00(0.00- \\
4.00)\end{array}$ & $3.00(1.00-4.25)$ & 0.160 \\
\hline $\mathrm{AlS}_{\text {face, }}$ median (IQR) & $0.00(0.00-0.00)$ & $\begin{array}{l}0.00(0.00- \\
0.25)\end{array}$ & $0.00(0.00-0.25)$ & 0.985 \\
\hline $\mathrm{AIS}_{\text {thorax }}$, median (IQR) & $3.00(3.00-3.75)$ & $3.00(2.75-3.00)$ & $3.00(3.00-4.00)$ & 0.056 \\
\hline $\mathrm{AlS}_{\text {abdomen}}$, median (IQR) & $2.00(0.00-2.75)$ & $2.00(0.00-3.25)$ & $2.00(0.00-2.25)$ & 0.829 \\
\hline $\mathrm{AIS}_{\text {extremitiy, }}$ median (IQR) & $2.00(0.00-3.00)$ & $2.00(2.00-3.00)$ & $2.00(0.00-3.00)$ & 0.218 \\
\hline $\begin{array}{l}\text { APACHE II score admission, } \\
\text { median (IQR) }\end{array}$ & $\begin{array}{l}9.00(3.00- \\
12.00)\end{array}$ & $5.50(3.00-8.25)$ & $\begin{array}{l}9.00(7.00- \\
11.00)\end{array}$ & 0.005 \\
\hline $\begin{array}{l}\text { SOFA score admission, median } \\
\text { (IQR) }\end{array}$ & $1.00(0.00-4.50)$ & $\begin{array}{l}1.00(0.00- \\
1.25)\end{array}$ & $2.00(2.00-4.00)$ & $<0.001$ \\
\hline Days in hospital & $\begin{array}{l}40.00(29.00- \\
66.75)\end{array}$ & $\begin{array}{l}44.00(25.50- \\
78.25)\end{array}$ & $\begin{array}{l}37.00(29.75- \\
66.25)\end{array}$ & 0.967 \\
\hline Days on ICU & $\begin{array}{l}0.00(0.00- \\
15.50)\end{array}$ & $\begin{array}{l}0.00(0.00- \\
1.25)\end{array}$ & $\begin{array}{l}13.00(0.00- \\
27.75)\end{array}$ & 0.003 \\
\hline \multicolumn{5}{|l|}{ Source of infection, n (\%) } \\
\hline Pulmonary & $12(30.0 \%)$ & - & $12(54.5 \%)$ & \\
\hline Urinary & $4(10.0 \%)$ & - & $4(18.2 \%)$ & \\
\hline Bloodstream & $1(2.5 \%)$ & - & $1(4.5 \%)$ & \\
\hline Wound & $4(10.0 \%)$ & - & $4(18.2 \%)$ & \\
\hline \multicolumn{5}{|l|}{ Pathogens, n (\%) } \\
\hline Gram-negative & $9(22.5 \%)$ & - & 9 (40.9\%) & \\
\hline
\end{tabular}




\begin{tabular}{|c|c|c|c|c|}
\hline Variables & Total $(n=40)$ & $\begin{array}{l}\text { Non-sepsis } \\
(n=18)\end{array}$ & Sepsis $(n=22)$ & $\begin{array}{l}P \\
\text { value }\end{array}$ \\
\hline Gram-positive & $5(12.5 \%)$ & - & $5(22.7 \%)$ & \\
\hline Fungi & $2(5.0 \%)$ & - & $2(9.1 \%)$ & \\
\hline $\begin{array}{l}\text { Mixed Gram negative and } \\
\text { positive }\end{array}$ & $5(12.5 \%)$ & - & $5(22.7 \%)$ & \\
\hline
\end{tabular}

\section{Dynamic Changes In Inflammatory Biomarkers In Trauma Patients}

We first characterized the kinetic changes in inflammatory biomarkers in the plasma of trauma patients. As shown in Figure 1 and Table S1, the plasma levels of six cytokines, including IL-6, IL-10, IP-10, sCD40L, GRO, and G-CSF, were significantly changed among the different timepoints (days 1, 3, 5, 7, and 14) in all trauma patients. Among them, IP-10, SCD40L, and GRO were significantly elevated with the progression of trauma, and the other three cytokines were significantly decreased during the disease course. For example, there was a significantly higher level of IP-10 on day 14 compared with day $1(p=0.005)$ and day 3 ( $p=0.009)$. The levels of IL-8, IL-15, TNF-a, MCP-1, MDC, eotaxin, MIP-1 $\beta$, GM-CSF, and FGF-2 showed no significant changes among the different timepoints after trauma.

\section{Time Course Of Cytokine Biomarkers As Related To Sepsis}

To delineate sterile inflammation from sepsis, we evaluated the association between the levels of cytokines and traumatic sepsis using Wilcoxon's rank sum test analysis (Figure 2 and Table S2). The levels of IL-6 (days 3 and 14), IL-10 (days 1 and 3), IL-15 (days 3, 5, and 14), G-CSF (days 3, 5, and 7) and FGF-2 (days 3) in the sepsis patients were significantly decreased compared to those in the nonsepsis controls. However, the levels of MDC (days 7 and 14), GRO (days 3 and 14), and SCD40L (days 14) in the sepsis patients showed significant increases compared with those of the nonsepsis controls. Other cytokine levels showed no significant difference between the two groups in the first 14 days.

To further corroborate the predictive ability of the eight cytokines with regard to the status of sepsis, we employed univariate binary logistic regression with ROC curve analysis. Eight cytokines were able to predict septic course at day 3 after trauma with an AUC ranging between 0.69 and $0.85(P<0.05)$. Combination of the eight cytokines demonstrated even higher AUCs of up to 0.90 (Figure 3 and Table S3). At day 3 after trauma, a remarkable increase (by 5\%) was demonstrated when combining the eight cytokines. Giving equal weight to both sensitivity and specificity, we selected -1.19 for the combined eight markers as a cut-off value for being able to predict yet clinically unapparent septic course at day 3 after trauma (sensitivity/specificity: $83 \% / 91 \%$ ). 


\section{Event-related Analysis Of Inflammatory Biomarkers}

To further elucidate the interaction between time and disease status, we rearranged the individual biomarker courses according to their timepoint of occurrence and used the grand median for patients with an uneventful course as a reference line. This event-related analysis (Figure 4) demonstrated that serum G-CSF levels had a 1.5-fold increase within 72 hours and a 3-fold increase within 48 hours before clinically apparent sepsis with subsequent initiation of therapy. In contrast, IL-10 levels appeared 4-fold higher within 72 hours and 1.5-fold higher before sepsis was clinically overt. IL-6 serum levels showed a circa 1.5-fold increase in patients with septic complications within 72 hours before being clinically overt. In the same time frame, FGF-2 decreased 2-fold in patients with sepsis. Eventually, serum levels of MDC, SCD40L, GRO, and IL-15 demonstrated unspecific undulations within 72 hours before diagnosis of the corresponding event.

\section{Discussion}

This study investigated the time course of 15 cytokines, including both pro- and anti-inflammatory cytokines and chemokines, in a cohort of 40 patients who experienced severe trauma with regard to the incidence of septic complications within the initial 14 days after trauma. As expected, the levels of five cytokines, including IL-6, IL-10, sCD40L, GRO, and G-CSF, were significantly interactional between time and the presence of sepsis, which was indicated by a significantly steeper change in plasma levels in patients with sepsis as opposed to those without sepsis. This interaction suggests that, besides a cut-off value, the time-related kinetics of cytokine biomarkers has a crucial role in the preclinical identification of septic patients.

When combining these cytokines, the ROC curve analysis achieved even higher AUCs of up to 0.90 at day 3. On the one hand, the increase in accuracy was favorable; on the other hand, day 3 after trauma demonstrated a remarkable increase (by $5 \%$ ) when combining these biomarkers. These data suggest that combining multiple cytokines might be advantageous, especially in the early phase after trauma. However, it is to be noted that standard ROC curve analysis represents a theoretical model, which does not account for time dependency of an event. This limitation of considering an event (i.e., sepsis) status for an individual as fixed over time is often encountered in biomarker studies and demands cautious data interpretation. Therefore, the event-related analysis as shown in Figure 4 compensates for that and better reflects the predictive strength of a biomarker. Against this background, IL-6, IL-10, G-CSF, and FGF-2 have excellent predictive ability for septic progression.

The ultimate goal in sepsis biomarker research, however, is the development of a score that is useful for sepsis prediction, diagnosis, and risk stratification. With the availability of systems biology approaches, such a sepsis score may ideally comprise a combination of several biomarkers, including genomic, transcriptomic, proteomic, and metabolomic candidates, as well as clinical parameters. Examples of preliminary scores in different sepsis cohorts include the combination of clinical and transcriptomic markers, metabolic and protein-mediator profiling, or the combination of biomarkers with established 
clinical scores $[7,15,16]$. In our opinion, some cytokines are also very promising candidates to be included in a sepsis score in general and in particular for discrimination of septic complications in patients with underlying systemic inflammation as indicated by the results of the present study. Nevertheless, based on the experience of previous studies [17], the development of a multibiomarker score for predicting and diagnosing sepsis represents an intricate endeavor. The implementation of novel fields, such as bioinformatics, computational biology, and machine learning, into sepsis research might open up new possibilities to adequately reflect the complexity and dynamics of sepsis for development of a valid and reliable diagnostic tool in the future.

As early decision making with the initiation of therapy is key to survival, especially in trauma, inflammatory biomarkers are urgently needed, aiming at the preclinical detection of deterioration [18]. In contrast, this patient population has some major methodological advantages in regard to testing novel biomarkers compared to other critically ill patients, making up for some key limitations of previous studies as follows: (A) Prophylactic administration of antibiotics, which is generally considered a major incommoding confounder in sepsis-related biomarker research, is strongly avoided in patients with blunt trauma injuries due to resistance breeding. (B) The trauma victim is mostly young to middle-aged with no or few comorbidities, thus greatly diminishing confounding effects. (C) The ICU stay of severe trauma victims is usually prolonged. Therefore, detailed monitoring and documentation of clinical and laboratory parameters are valuable prerequisites to retrospectively identify rapid changes in the conditions of patients. (D) Trauma victims are thought to be more susceptible to infectious complications than any other patient population, allowing for statistically balanced juxtaposition of septic versus nonseptic

patients. (E) Trauma represents a rather homogenous entity with directly measurable trauma severity (i.e., ISS and AIS), which can simply be adjusted for statistical analyses.

Elucidating the role of cytokines in severe trauma patients with respect to the occurrence of sepsis, the present study represents a crucial step on the way to testing for potentially helpful biomarkers for early sepsis prediction and detection. In this context, the present study needs to be considered as hypothesis generating and awaiting confirmation in future clinical trials, since there are some limitations. Given the relatively small number of patients included and the single center design of our study, there is no external validation of our data, which has to be addressed in further studies. Likewise, measurement of biomarkers and assessment of clinical parameters were performed only once per 48 hours, neglecting potential alterations between these intervals. Moreover, the influence of the type of infection and of repetitive surgical tissue damage on cytokine serum levels need to be clarified in further studies. We believe that these findings will have implications for the management of patients with trauma.

\section{Conclusions}

Cytokine profiles demonstrate high discriminatory ability to timely identify evolving sepsis in patients with severe blunt trauma. These abrupt changes allow sepsis detection up to 72 hours before clinically overt deterioration. Affordable cytokine bedside measurement might enable earlier treatment of sepsis with reduced morbidity and mortality and subsequently reduce costs for trauma patients in the future. 


\section{Abbreviations}

ISS, Injury Severity Score; CRP, C-reaction protein; PCT, procalcitonin; TNF, tumor necrosis factor; IL, interleukin; MIP, macrophage inflammatory protein; HSP, heat shock protein; MMP, matrix metalloprotease; SIRS, systemic inflammatory response syndrome; MEWS, modified early warning score; SOFA, sequential organ failure assessment; MDC, macrophage derived chemokine; IP, interferon-inducible protein; GRO, growth regulated oncogene; MCP, monocyte chemotactic protein ; FGF, fibroblast growth factor; G-CSF, granulocyte colony-stimulating factor; GM-CSF, granulocyte macrophage colony-stimulating factor; IQR, interquartile range; SD, standard deviation; ROC, receiver operating characteristics; $A U C$, area under curve; $\mathrm{Cl}$, confidence interval; ICU, intensive care unit.

\section{Declarations}

\section{Ethics approval and consent to participate}

The research was approved by the Institutional Ethics Review Board of the Army Medical University (No. TMMU2012009). The study was performed in accordance with the Helsinki Declaration of 1964, and its later amendments. Informed consent of all patients was obtained from the patients or their relatives.

\section{Consent for publication}

Not applicable

\section{Availability of data and material}

The datasets presented in this study can be found in online repositories. The names of the repository/repositories and accession number(s) can be found in the article/Supplementary Material. The datasets used and/or analyzed during the current study are available from the corresponding author on reasonable request.

\section{Competing interests}

All authors declare that they have no competing interests. All authors read and approved the final manuscript.

\section{Funding}

This work was supported by National Natural Science Foundation of China (81971830); Research project of clinical and military medical, Army Medical University (2019XLC3048 and 2019CXJSC006); and Open Project Program of the State Key Laboratory of Trauma, Burn and Combined Injury (SKLKF201802).

\section{Authors' contributions}


JW and D-LW were the main researchers for this study and contributed to write this manuscript. SZ, JD, LC, D-YD and L-YZ were involved in the collecting of blood samples. J-HS, H-CZ and LZ did the technical work. JD, J-XJ, and A-QZ planned the study, wrote the protocol and was involved in the data analyses and revised the manuscript.

\section{Acknowledgements}

We acknowledge Dr. Xiao Wang, General Hospital of Western Theater Command, for his technical help. Dr. Dong-po Jiang, Army Medical University, and Dr. JunYang, Chongqing Emergency Medical Center, for collection of the blood samples. This manuscript has been released as a pre-print at Research Square. Available at: https://www.researchsquare.com/article/rs-84088/v1 (Accessed Oct 02, 2020; updated Feb 20, 2021).

\section{References}

1. Rhodes A, Evans LE, Alhazzani W, Levy MM, Antonelli M, Ferrer R, Kumar A, Sevransky JE, Sprung CL, Nunnally ME et al: Surviving Sepsis Campaign: International Guidelines for Management of Sepsis and Septic Shock: 2016. Intensive Care Med 2017, 43(3):304-377.

2. Singer M, Deutschman CS, Seymour CW, Shankar-Hari M, Annane D, Bauer M, Bellomo R, Bernard GR, Chiche JD, Coopersmith CM et al: The Third International Consensus Definitions for Sepsis and Septic Shock (Sepsis-3). JAMA 2016, 315(8):801-810.

3. Kim MH, Choi JH: An Update on Sepsis Biomarkers. Infect Chemother 2020, 52(1):1-18.

4. Stanski NL, Wong HR: Prognostic and predictive enrichment in sepsis. Nat Rev Nephrol 2020, 16(1):20-31.

5. Delano MJ, Ward PA: The immune system's role in sepsis progression, resolution, and long-term outcome. Immunol Rev 2016, 274(1):330-353.

6. D'Elia RV, Harrison K, Oyston PC, Lukaszewski RA, Clark GC: Targeting the "cytokine storm" for therapeutic benefit. Clin Vaccine Immunol 2013, 20(3):319-327.

7. Trzeciak A, Pietropaoli AP, Kim M: Biomarkers and Associated Immune Mechanisms for Early Detection and Therapeutic Management of Sepsis. Immune Netw 2020, 20(3):e23.

8. Muñoz B, Suárez-Sánchez R, Hernández-Hernández O, Franco-Cendejas R, Cortés $H$, Magaña JJ: From traditional biochemical signals to molecular markers for detection of sepsis after burn injuries. Burns 2019, 45(1):16-31.

9. Cahill LA, Joughin BA, Kwon WY, Itagaki K, Kirk CH, Shapiro NI, Otterbein LE, Yaffe MB, Lederer JA, Hauser CJ: Multiplexed Plasma Immune Mediator Signatures Can Differentiate Sepsis From NonInfective SIRS: American Surgical Association 2020 Annual Meeting Paper. Ann Surg 2020, 272(4):604-610.

10. Rangel-Frausto MS, Pittet D, Costigan M, Hwang T, Davis CS, Wenzel RP: The natural history of the systemic inflammatory response syndrome (SIRS). A prospective study. JAMA 1995, 273(2):117-123. 
11. Subbe CP, Slater A, Menon D, Gemmell L: Validation of physiological scoring systems in the accident and emergency department. Emerg Med J 2006, 23(11):841-845.

12. Vincent JL, Moreno R, Takala J, Willatts S, De Mendonca A, Bruining H, Reinhart CK, Suter PM, Thijs LG: The SOFA (Sepsis-related Organ Failure Assessment) score to describe organ dysfunction/failure. Intensive Care Medicine 1996, 22(7):707-710.

13. The Abbreviated Injury Scale: Association for the Advancement of Automotive Medicine, Barrington. 2005(Illinois).

14. Zhang AQ, Gu W, Zeng L, Zhang LY, Du DY, Zhang M, Hao J, Yue CL, Jiang J: Genetic Variants of microRNA Sequences and Susceptibility to Sepsis in Patients With Major Blunt Trauma. Ann Surg 2015, 261(1):189-196.

15. Wang HE, Donnelly JP, Yende S, Levitan EB, Shapiro NI, Dai Y, Zhao H, Heiss G, Odden M, Newman A et al: Validation of the REGARDS Severe Sepsis Risk Score. J Clin Med 2018, 7(12):536.

16. Shankar-Hari M, Datta D, Wilson J, Assi V, Stephen J, Weir CJ, Rennie J, Antonelli J, Bateman A, Felton JM et al: Early PREdiction of sepsis using leukocyte surface biomarkers: the ExPRES-sepsis cohort study. Intensive Care Med 2018, 44(11):1836-1848.

17. Lippi G: Sepsis biomarkers: past, present and future. Clin Chem Lab Med 2019, 57(9):1281-1283.

18. Peters van Ton AM, Kox M, Abdo WF, Pickkers P: Precision Immunotherapy for Sepsis. Front Immunol 2018, 9:1926.

\section{Figures}



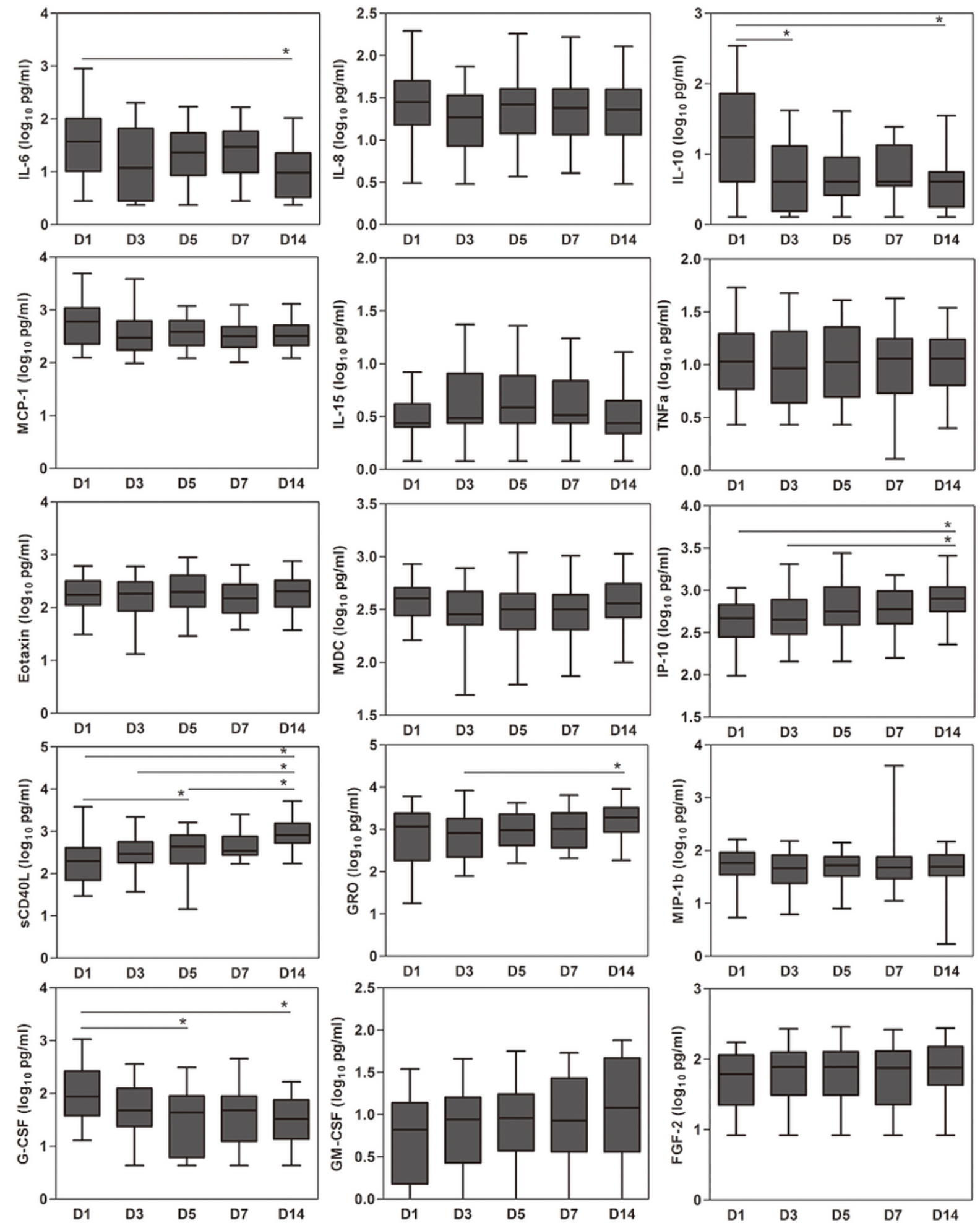

Figure 1

Dynamic changes in the levels of 15 cytokines among blunt trauma patients. The cytokine values were transformed to common logarithm values to normalize the distribution of the data. The boxes indicate the lower and upper quartiles, the central line is the median, and the ends of the whiskers represent the maximum and minimum values. Asterisks indicate a significant difference between different timepoints $(P<0.05)$. Interleukin, IL; monocyte chemotactic protein, MCP; tumor necrosis factor, TNF; macrophage 
derived chemokine, MDC; soluble CD40 ligand, SCD40L; growth regulated oncogene, GRO; macrophage inflammatory protein, MIP; interferon-inducible protein, IP; granulocyte colony-stimulating factor, G-CSF; granulocyte macrophage colony-stimulating factor, GM-CSF; fibroblast growth factor, FGF.
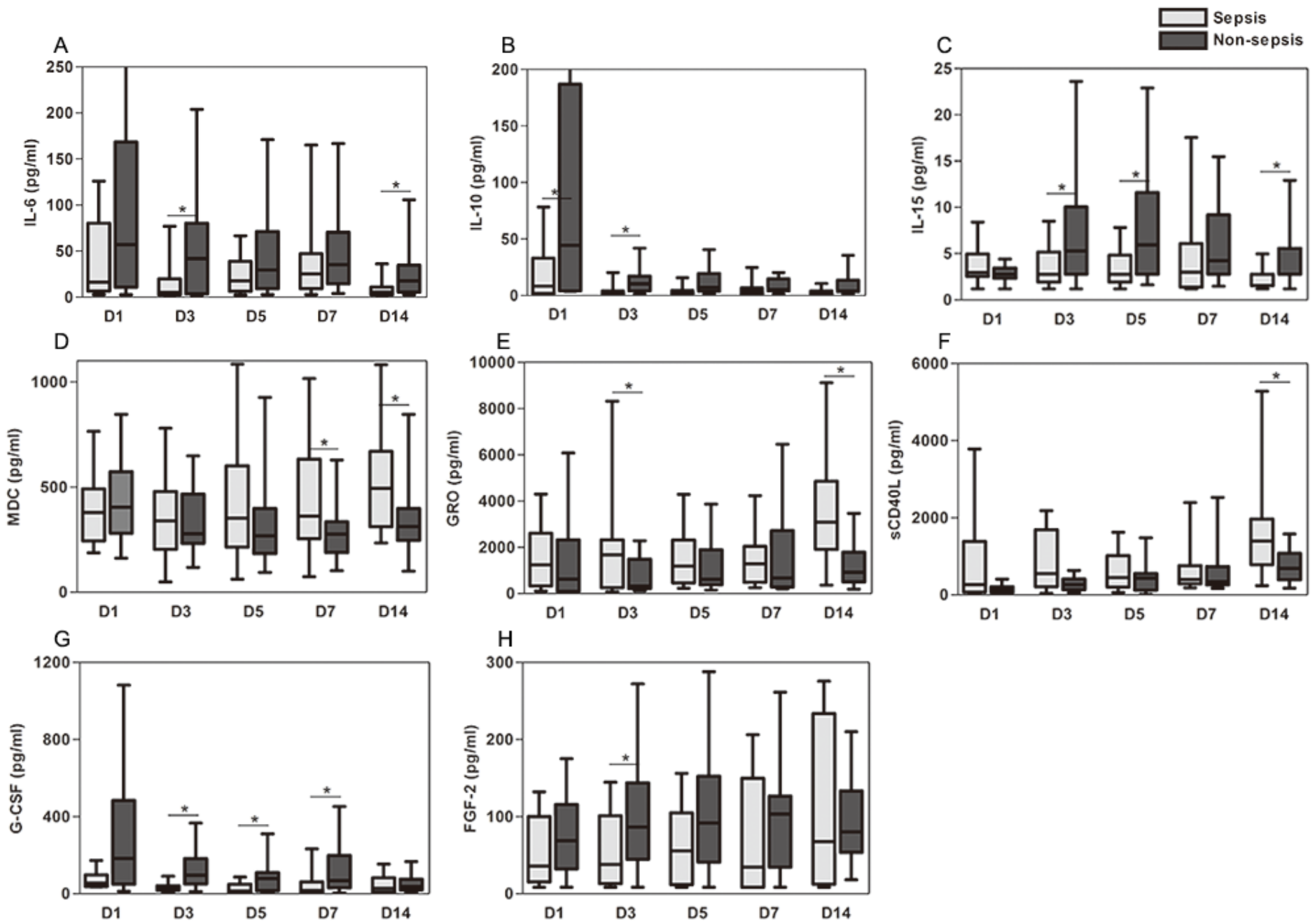

Figure 2

Levels of cytokines in sepsis patients and nonsepsis patients. (A) Interleukin (IL)-6, (B) IL-10, (C) IL-15, (D) macrophage derived chemokine (MDC), (E) growth regulated oncogene (GRO), (F) soluble CD40 ligand (sCD40L), (G) granulocyte colony-stimulating factor (G-CSF), and (H) fibroblast growth factor (FGF)-2 levels on day $1,3,5,7$, and 14 . The boxes indicate the lower and upper quartiles, the central line is the median, and the ends of the whiskers represent the maximum and minimum values. Asterisks indicate a significant difference between sepsis and nonsepsis patients $(P<0.05)$. 


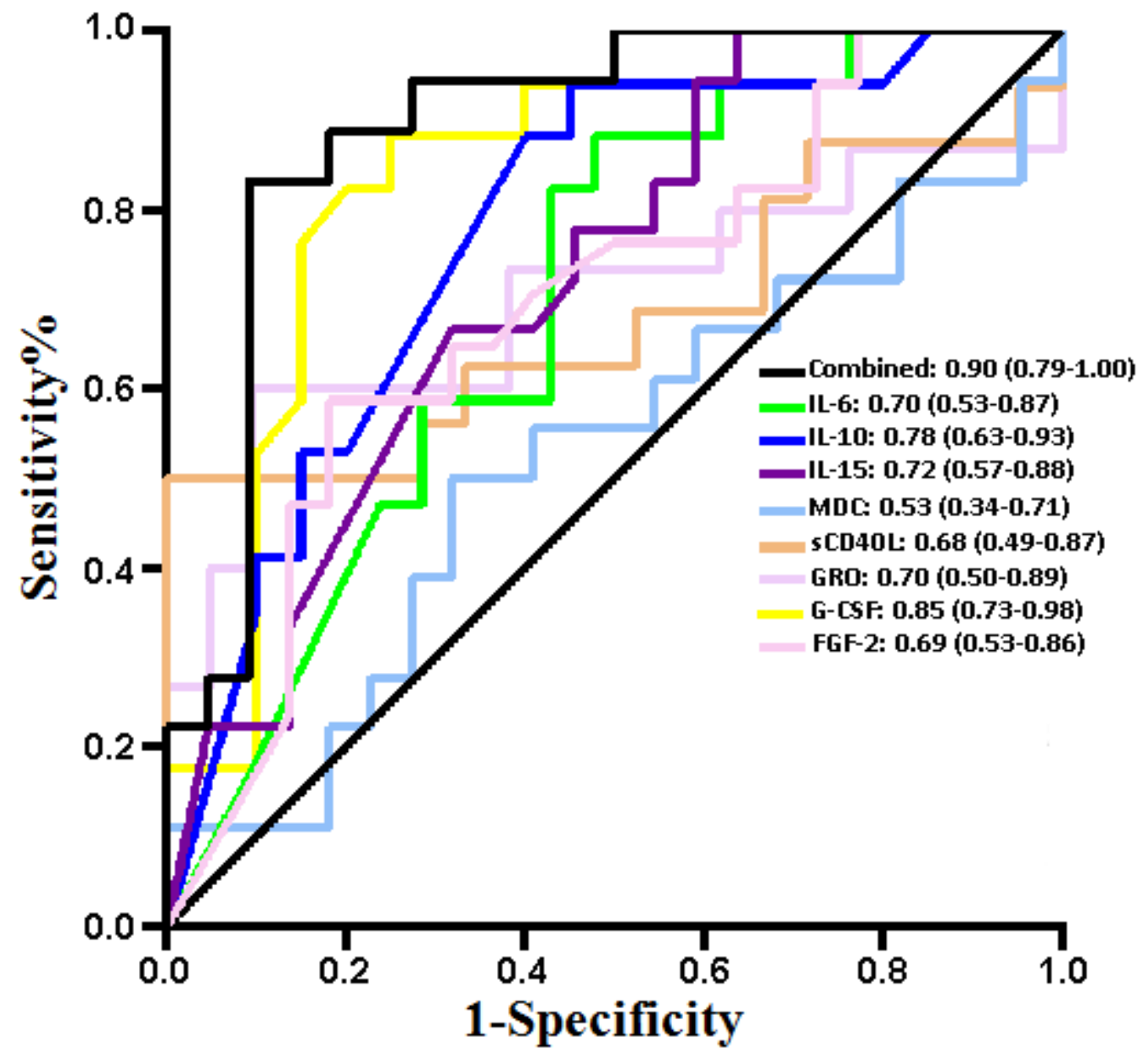

Figure 3

ROC curve analysis using the cytokines in the trauma patients with sepsis. AUC and $95 \% \mathrm{Cl}$ demonstrating the respective and combined six cytokines to predict sepsis on day 3 after trauma, with AUC of up to 0.90 . AUC, area under the curve; ROC, receiver operating characteristic; interleukin, IL; macrophage derived chemokine, MDC; growth regulated oncogene, GRO; soluble CD40 ligand, CD40L; granulocyte colony-stimulating factor, G-CSF; fibroblast growth factor, FGF. 

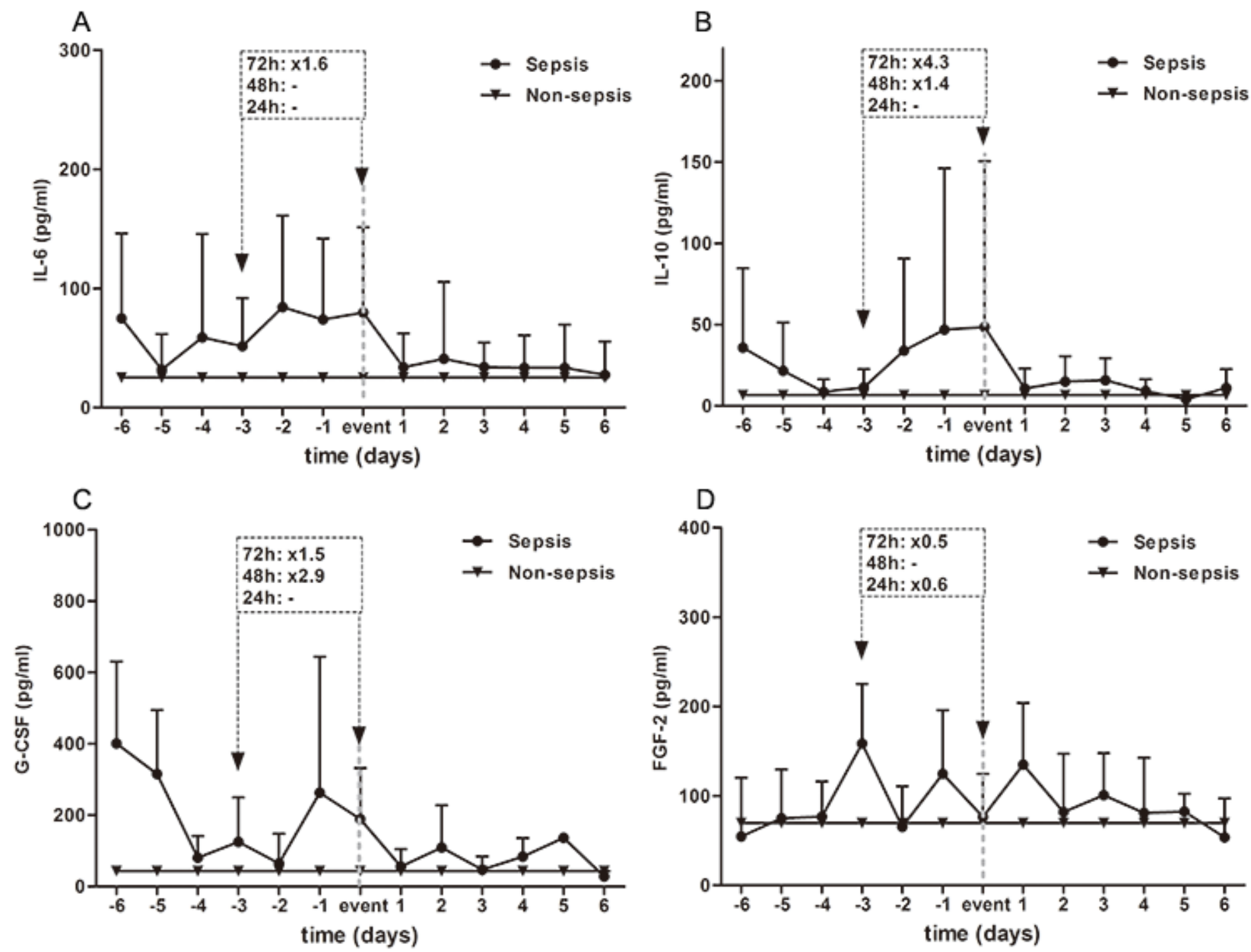

Figure 4

Event-related depiction of cytokines. The boxes in gray contain the relative cytokine increase for sepsis at 72 hours, 48 hours, and 24 hours. (A) Interleukin (IL)-6, (B) IL-10, (C) granulocyte colony-stimulating factor (G-CSF), and (D) fibroblast growth factor (FGF)-2.

\section{Supplementary Files}

This is a list of supplementary files associated with this preprint. Click to download.

- SupplementaryMaterial.doc 